\title{
MODELLING OF E-CLOUD BUILD-UP IN GROOVED VACUUM CHAMBERS USING POSINST**
}

\author{
M. Venturini ${ }^{\dagger}$, M. Furman, and J-L. Vay (LBNL, Berkeley, California), \\ M. Pivi (SLAC, Menlo Park, California)
}

\begin{abstract}
Use of grooved vacuum chambers have been suggested as a way to limit electron cloud accumulation in the ILCDR. We report on simulations carried out using an augmented version of POSINST, accounting for e-cloud dynamics in the presence of grooves, and make contact with previous estimates of an effective secondary electron yield for grooved surfaces.
\end{abstract}

\section{INTRODUCTION}

Electron cloud accumulation and related instabilities are of serious concern for the International Linear Collider (ILC) positron damping ring (DR). Surface coating, scrubbing, and conditioning are known methods to mitigate electron build-up but may be insufficient. It is believed that to achieve the baseline specifications for the machine performance [1] effective techniques to suppress electron cloud build-up beyond levels currently demonstrated will have to be developed. As a consequence 'non-traditional' techniques, including clearing electrodes and grooved vacuum chambers, are being actively investigated.

Analytical and numerical modelling of the interaction of electrons with grooved surfaces have indicated the effectiveness of this technique and accelerator-based experiments to confirm these results are planned or already underway. Previous simulations [2, 3, 4] so far have generally aimed at determining an effective secondary electron yield (SEY) by considering a beam of monochromatic electrons (primary particles) impinging on the grooved surface and keeping track of the electrons (secondary particles) emerging from the groove regions - a setting typical of laboratory bench measurements where an effective SEY can easily be determined as a function of the energy of the primary electron beam. In the work described here we are interested in a direct characterization of the electron cloud build-up in the vacuum chamber of an operating accelerator in the presence of both the driving beam and space-charge from the electrons. This will be useful for a closer comparison between current e-cloud modelling and accelerator-based measurements.

* Work supported by DOE, Contract no. DE-AC02-OSCH11231. Also as LBNL-63110.

$\dagger$ mventurini@1bl.gov

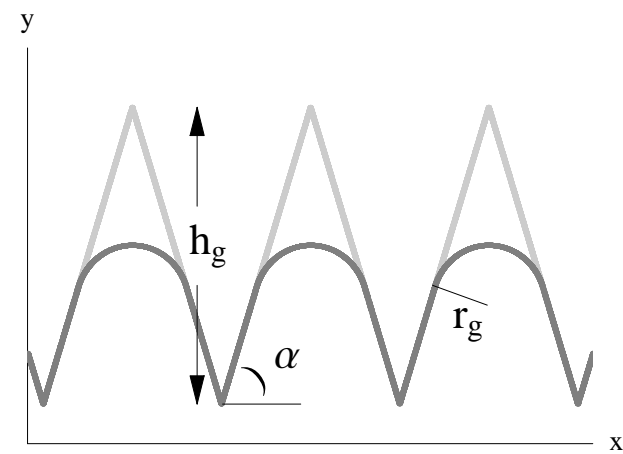

Figure 1: Triangular grooves with sharp tips are characterized by the steepness angle $\alpha$ and height $h_{g}$. Grooves with rounded tips have the additional parameter $r_{g}$, the radius of the groove tip. The interior of the chamber is on the top side.

\section{IMPLEMENTATION AND SIMULATIONS}

We carried out our work by augmenting the current version of the code POSINST to include the option to follow the electron dynamics in the presence of grooves. Electronsurface collisions and secondary electron production following those collisions are modelled using the modules already built in POSINST $[5,6]$. At present we have a provision to simulate rectangular cross-section vacuum chambers with triangular grooves located on the top and bottom sides - closely reproducing the configuration of a proposed e-cloud experiment at PEP-II. The steepness angle $\alpha$ of the triangular grooves as well their height (see Fig. 1) are input parameters controlled by the user. An option to include rounding of the groove tips has also been implemented. Space charge from the electrons is included in the model. However, at present the electric field lines are terminated on a hypothetical smooth surface immediately behind the grooves thus neglecting possible field enhancement by the groove tips.

Grooves reduce the effective SEY by increasing the probability that immediately after production secondary electrons may be rapidly reabsorbed through wall collisions and therefore prevented from contributing to multipacting. The effectiveness of the grooves strongly depends on the geometry. For triangular grooves the existence of a critical angle for effective suppression of the electron cloud can be clearly extracted from Fig. 2 and 3. Fig. 3 shows the maximum linear electron density accumulated during the single passage of a 111-positron bunch train through one of 


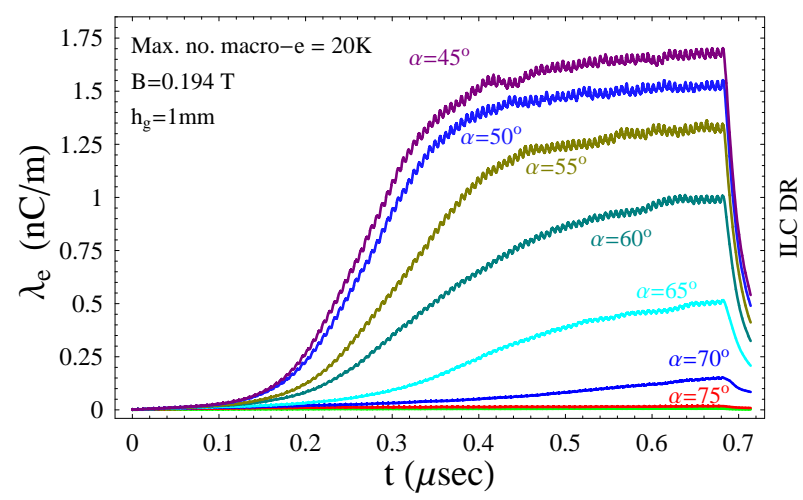

Figure 2: E-cloud accumulation during passage of a $0.68 \mu \mathrm{sec}$ bunch train for various steepness angles of triangular (sharp edge) grooves.

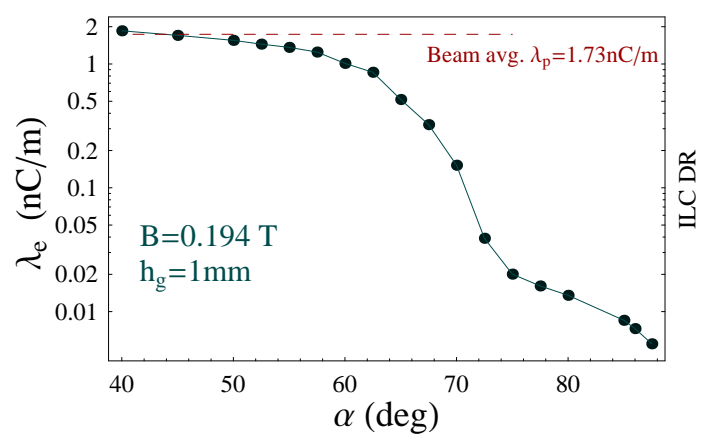

Figure 3: Maximum linear density of electrons accumulated during a passage of a train of positron bunches in a ILC damping ring dipole vs. steepness angle $\alpha$ of triangular grooves (with sharp edges). Al chamber with $\delta_{\max }=1.75$.

the ILC DR dipoles as a function of the triangular grooves steepness angle $\alpha$. All the calculations reported in this paper are for the ILC-DR dipoles. The bunch train is $0.68 \mu \mathrm{s}$ long, for a $6.1 \mathrm{~ns}$ separation between bunches. The bunches have a population of $2 \times 10^{10}$ and sizes $\sigma_{x}=0.62 \mathrm{~mm}$, $\sigma_{y}=8 \mu \mathrm{m}, \sigma_{z}=6 \mathrm{~mm}$ (this is smaller than the current baseline value $\sigma_{z}=9 \mathrm{~mm}$ ). The magnetic field in the dipoles is about $0.2 \mathrm{~T}$. The calculation is for grooves height $h_{g}=1 \mathrm{~mm}$ and the model of SEY adopted was that of Al, with maximum SEY set to $\delta_{\max }=1.75$.

A drop in electron density by about two orders of magnitude compared to the smooth-chamber case is seen to occur for steepness angle $\alpha$ larger than $75^{\circ}$. For shallower angles the electron accumulation is increasingly larger, approaching and in fact slightly overtaking the electron cloud density for a smooth surface when $\alpha<45^{\circ}$. This latter behavior is not implausible. It is a basic property of the model employed in the calculation that the SEY is minimum for electrons hitting the surface at a normal incidence. At a smaller $\alpha$ the grooves become ineffective at capturing the secondaries and the effective SEY may become larger if on

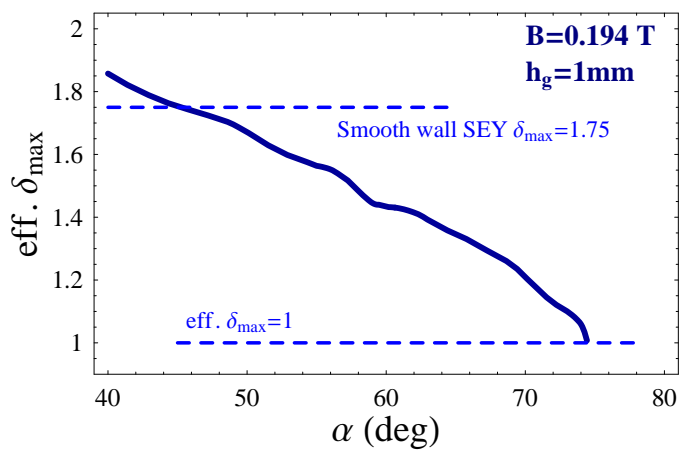

Figure 4: Effective SEY as a function of the steepness angle $\alpha$ as derived from the simulation of e-cloud build-up.

average the primary electrons hit the surface off the local normal.

To make contact with previous studies we extracted an effective max SEY from our data by making comparison with the electron density we would obtain in a smooth chamber as we vary the value of $\delta_{\max }$ for the smooth surface. An effective yield corresponding to a given $\alpha$ is then defined as that $\delta_{\max }$ producing the same maximum e-cloud accumulation in a smooth chamber during the passage of the same train of positron bunches. The result is shown in Fig. 4 where the effective max. SEY is plotted as a function of the steepness angle $\alpha$. The curve is reasonably smooth and again indicates $\alpha \simeq 75^{\circ}$ as the critical angle where the effective secondary yield crosses into values smaller than unity corresponding to effective electron cloud suppression.

Our results are substantially consistent with calculations reported in [2], where for the same groove geometry (and same maximum SEY for the smooth surfaces) an effective secondary yield as a function of energy is found to remain $<1$ for angles just above $\alpha \simeq 70^{\circ}$. Both the present and Wang et al.'s findings are somewhat less pessimistic than those obtained by W. Bruns [4], which indicate that an angle $\alpha=75^{\circ}$ would still yield an effective SEY larger than unity; $\alpha=75^{\circ}$ was the largest steepness angle reported in [4] but a rough extrapolation from the data shown would appear to predict a noticeably larger critical angle for a $\mathrm{SEY}<1$.

There have been speculations that these discrepancies in the results could perhaps be ascribed to differences in the SEY model at low electron energies. While in the model used by W. Bruns $\delta(E)$ is unity at zero energy [7, 8] (and displays a local minimum at low energy ) in the POSINST model (and possibly in L. Wang's calculations [2]), the same limit is $\delta(0) \simeq 0.5$ [6]. It is not unlikely that the capturing properties of the grooves may be sensitive to the details of the yield curve at small energy but we have yet to run simulations to test this supposition.

A systematic study of the dependence of the electron cloud build- up for a given geometry of the grooved surface on the strength of the magnetic field is shown in Fig. 5, where the magnetic field values for the ILC DRs dipoles 


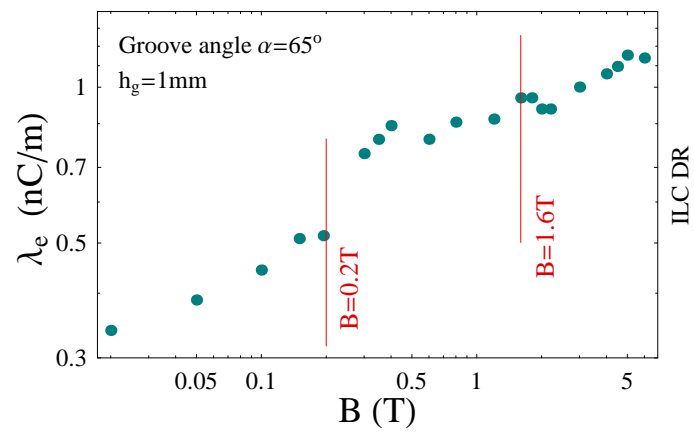

Figure 5: Max. accumulated e-cloud as a function of the magnetic field for a given grooved surface geometry.

and wigglers have been highlighted (but in both cases the calculation is done for a dipole with uniform B-field). In general a larger magnetic field makes the triangular grooving less effective. The various levels of e-cloud accumulation shown in the picture also reflect a larger number of primary photo-electrons produced at a larger B-field.

A drawback of grooving the inner surface of a vacuum chamber is an enhancement of the resistive wall impedance [2]. A possible remedy is to introduce some rounding at the edges of the triangular grooves hence reducing the field enhancement caused by sharp boundaries. Rounding the tips, however, can be expected to degrade the e-cloud suppression property of the grooves (electrons impinging on the rounded surface on the groove tips are less likely to be recaptured) and its modelling should be included in the simulations. Moreover, studying the effect of the tip rounding would be important in setting acceptable tolerances on the machining of the grooves. The present implementation in POSINST only allows for the rounding of the triangular groove edges facing the inner side of the chamber (see Fig. 1).

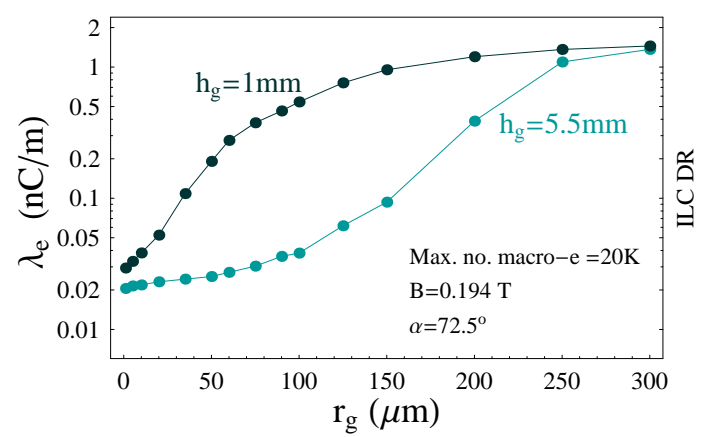

Figure 6: Max. accumulated e-cloud for fixed $\alpha$, as a function of the groove tip radius $r_{g}$ for two choices of groove height.

Our simulations confirm the expected degradation of ecloud suppression by the rounding of the tips. In Fig. 6 the maximum e-cloud density accumulated during the passage

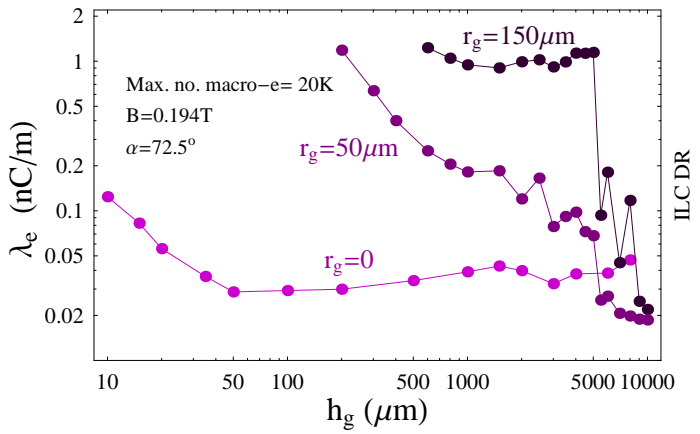

Figure 7: Max. accumulated e-cloud, for fixed $\alpha$, as a function of the groove hight $h_{g}$ for three choices of the grove tip radius $r_{g}$.

of a bunch train is reported as a function of the tip radius $r_{g}$ for a given steepness angle $\alpha$ and two choices of the groove height $h_{g}$. For $h_{g}=1 \mathrm{~mm}$ we observe a substantial increase of the e-cloud density for $r_{g} \simeq 50 \mu \mathrm{m}$ or larger. The simulations suggest that one way to recover the suppression of electrons is to deepen the grooves. For a fixed steepness angle this results into a smaller number of grooves per unit-length and a smaller ratio between rounded tip surface and groove aperture. The same picture shows that a $5.5 \mathrm{~mm}$ groove height could withstand a rounding radius up to $100 \mu \mathrm{m}$ without a substantial degradation of the grooves effectiveness.

Finally, a study of the dependence of electron accumulation as a function of the groove height for three choices of the rounding tip radius $r_{g}$ is reported in Fig. 7 confirming that sufficiently deep grooves can suppress the electron cloud build-up significantly regardless of the tip radius.

In conclusion, we have enhanced the current version of POSINST to allow for modelling of the electron cloud dynamics in grooved vacuum chambers. We expect this to be a useful feature in particular for simulation of acceleratorbased experimental measurements presently under consideration.

Interesting conversations with W. Bruns and R. Cimino, are gratefully acknowledged.

\section{REFERENCES}

[1] M. Pivi et al., Simulations of the Electron Cloud for Various Configurations of a Damping Ring , EPAC06 proceedings (2006).

[2] L. Wang et al., NIM-A 571 (2007) p. 588.

[3] G. Stupakov and M. Pivi, SLAC-TN-04-045, SLAC (2004).

[4] G. Rumolo and F. Zimmermann, "LC e-Cloud Activities at CERN", talk presented at the ILC-DR 2006 Workshop, Cornell (2006).

[5] M. A. Furman, "The electron-cloud effect in the arcs of the LHC,' LBNL- c 41482/CBP Note 247/LHC Project Report 180, May 20, (1998).

[6] M. A. Furman and M. T. F. Pivi, LBNL-49771, Phys Rev. ST-AB 5124404 (2003); and LBNL-52807. 
[7] R. Cimino et al., PRL 93014801 (2004).

[8] G. Bellodi, CARE-HHH 2004 Proceedings (2004).
This document was prepared as an account of work sponsored by the United States Government. While this document is believed to contain correct information, neither the United States Government nor any agency thereof, nor The Regents of the University of California, nor any of their employees, makes any warranty, express or implied, or assumes any legal responsibility for the accuracy, completeness, or usefulness of any information, apparatus, product, or process disclosed, or represents that its use would not infringe privately owned rights. Reference herein to any specific commercial product, process, or service by its trade name, trademark, manufacturer, or otherwise, does not necessarily constitute or imply its endorsement, recommendation, or favoring by the United States Government or any agency thereof, or The Regents of the University of California. The views and opinions of authors expressed herein do not necessarily state or reflect those of the United States Government or any agency thereof, or The Regents of the University of California.

Ernest Orlando Lawrence Berkeley National Laboratory is an equal opportunity employer. 